\title{
Breve análisis crítico de la sobreoferta y consumo de alimentos industrializados en México, antes y durante el COVID-19
}

\section{Administration of food services to communities from the nutritionist's approach.}

\author{
Edú Ortega Ibarra ${ }^{a}$, Ilse Haide Ortega Ibarra ${ }^{b}$ Edna Rodríguez-López ${ }^{c}$, Carmen Luis Pineda
}

\begin{abstract}
:
Mexico has experienced a demographic, epidemiological, and nutritional transition in recent decades. In the last 40 years, the Mexican diet has gone from being primarily fresh, with unprocessed local and regional foods, to ultra-processed foods. The food industry is responsible for producing large amounts of food that are consumed throughout the world, so there have been changes that have been extremely evident in the diet, these changes favor the appearance of chronic-degenerative diseases such as overweight and obesity According to data from ENSANUT 2018, the overweight figures for women is $36.6 \%$ while obesity is $40.2 \%$; for men it is $42.5 \%$ and $30.5 \%$ for overweight and obesity respectively, an increase being observed compared to 2012, among the factors that could influence these figures is the oversupply of industrialized foods, increasing their consumption and making them an important source of energy. The COVID-19 pandemic has generated changes in lifestyles and consequently in the food choices that are made.
\end{abstract}

Keywords:

Industrialized food, oversupply, food, COVID-19, Mexico

Resumen:

México ha experimentado las últimas décadas una transición demográfica, epidemiológica y nutricional. En los últimos 40 años, la dieta mexicana ha pasado de ser principalmente fresca, con alimentos locales y regionales sin procesar a alimentos ultraprocesados. La industria alimentaria es la encargada de producir grandes cantidades de alimentos que son consumidos en todo el mundo, por lo que se han registrado cambios que han sido sumamente evidentes en la alimentación, estos cambios favorecen la aparición de enfermedades crónico-degenerativas como sobrepeso y obesidad, de acuerdo con datos de la ENSANUT 2018 las cifras de sobrepeso para mujeres es de $36.6 \%$ mientras que de obesidad es de $40.2 \%$; para los hombres es de $42.5 \%$ y $30.5 \%$ para sobrepeso y obesidad respectivamente, observándose un incremento a comparación de 2012, dentro de los factores que pudieran influir en estas cifras es la sobreoferta de alimentos industrializados, aumentando su consumo y convirtiéndolos en una importante fuente de energía. La pandemia por COVID-19 ha generado cambios en los estilos de vida y por consecuente en la elección de alimentos que se realizan.

Palabras Clave:

Alimentos industrializados, sobreoferta, alimentos, COVID-19, México

\section{Introducción}

México ha experimentado las últimas décadas una transición demográfica, epidemiológica y nutricional debido al envejecimiento de su población, el incremento de las enfermedades crónicas no transmisibles, así como del consumo de alimentos y bebidas con un alto contenido de grasas saturadas, azúcares y sal. ${ }^{1}$

\footnotetext{
Universidad del Istmo, campus Juchitán de Zaragoza, Oaxaca. E.O.I.: https://orcid.org/0000-0002-6504-7366, Email eoi@bizendaa.unistmo.edu.mx Autor de Correspondencia.

Universidad del Istmo, campus Juchitán de Zaragoza, Oaxaca., https://orcid.org/0000-0002-1104-2949, Email: ihoi@bizendaa.unistmo.edu.mx

Universidad del Istmo, campus Juchitán de Zaragoza, Oaxaca. https://orcid.org/0000-0001-6688-8362, Email: eirl@bizendaa.unistmo.edu.mx

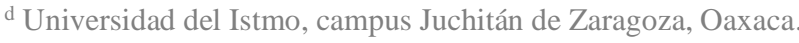


En los últimos 40 años, la dieta mexicana ha pasado de ser principalmente fresca, con alimentos locales y regionales sin procesar a alimentos ultra procesados, en este sentido, la industria alimentaria es la encargada de producir grandes cantidades de alimentos que son consumidos en todo el mundo, por lo que se han registrado cambios que han sido sumamente evidentes en la alimentación y que están relacionados con la gran modernización de dicha industria. Existe un notable incremento en la distribución y producción de los alimentos industrializados lo que conlleva a que las familias tengan un mayor acceso a este tipo de alimentos. ${ }^{2}$

Otros factores que han llevado a un cambio en la alimentación son: la desregulación del mercado, industrias de alimentos transnacionales, cadenas de comida rápida, mercadotecnia, urbanización, incremento de la proporción de mujeres que trabajan fuera de casa y el crecimiento económico. Estos cambios en los patrones de alimentación, aunado con la mayor disponibilidad de las familias mexicanas para adquirir estos alimentos altos en azúcares, grasas y sodio, han sido asociados a un mayor incremento del sobrepeso y obesidad.

El sobrepeso y la obesidad aumentan la aparición de enfermedades crónico-degenerativas como diabetes mellitus tipo II, hipertensión arterial, hipercolesterolemia, entre otras. Lo cual incrementa la morbimortalidad y disminución de calidad de vida en las familias. ${ }^{3}$ La amplia gama de alimentos industrializados ha llegado a modificar los patrones alimenticios en los mexicanos, debido a que existe una mayor preferencia al consumo de estos tipos de alimentos con altos aportes calóricos, elevados en azúcares simples y grasas saturadas aunado a una vida sedentaria, y falta de educación alimentaria puede favorecer al incremento de las enfermedades crónicodegenerativas. ${ }^{4}$

\section{¿QUÉ ES UN ALIMENTO INDUSTRIALIZADO?}

Los alimentos industrializados pueden ser definidos como aquellos que, al utilizar sustancias derivadas de los alimentos o los alimentos mismos, se obtienen nuevos productos a través de procesos de síntesis de sustancias, para generar un nuevo producto. Se ha demostrado que estos productos son poco saludables por lo que es sumamente importante contar con regulaciones que indiquen el contenido nutrimental de los mismos. ${ }^{5}$

La clasificación NOVA ha propuesto un enfoque para clasificar a los alimentos y bebidas en cuatro grupos de acuerdo a su nivel de procesamiento: a) no procesados o mínimamente procesados, b) ingredientes culinarios procesados, c) alimentos procesados y d) alimentos ultraprocesados. $^{6}$

NOVA define a los alimentos no procesados 0 mínimamente procesados como partes de plantas o alimentos que no se han sometido a ningún procesamiento industrial ni han sido alterados de manera en que se les agregue o introduzca cualquier sustancia, pero pueden implicar la eliminación de partes no comestibles o no deseadas de los alimentos.

Los ingredientes culinarios son sustancias extraídas y purificadas por la industria a partir de componentes alimentarios u obtenidos de la naturaleza con el fin de ser utilizados para preparar alimentos (por ej. Sal, azúcar, aceite). Los alimentos procesados son productos elaborados agregando azúcar, sal y otros ingredientes culinarios a los alimentos mínimamente procesados para incrementar su vida de anaquel y hacerlos más palatables.

Por último, los alimentos ultraprocesados son formulaciones industriales listas para ser consumidas que son fabricadas a partir de cinco o más ingredientes que comúnmente se utilizan en la industria como aceites, grasas, azúcar, caseína, lactosa, suero y gluten o ingredientes sintetizados a partir de fuentes orgánicas (aceites hidrogenados, maltodextrina, aislado de proteína de soya, jarabe de maíz), conservantes y aditivos (humectantes, emulsionantes, disolventes, agentes de carga, edulcorantes, colorantes y aromatizantes)

Clasificación de los alimentos industrializados con base a su nivel de procesamiento. ${ }^{7}$

\begin{tabular}{|c|c|c|}
\hline $\begin{array}{l}\text { Mínimamente } \\
\text { procesados }\end{array}$ & $\begin{array}{l}\text { Medianamente } \\
\text { procesados }\end{array}$ & $\begin{array}{l}\text { Ultra } \\
\text { procesados }\end{array}$ \\
\hline $\begin{array}{l}\text { Aquellos que no } \\
\text { son modificados } \\
\text { profundamente. } \\
\text { Esto quiere } \\
\text { decir que los } \\
\text { nutrientes, } \\
\text { consistencia o } \\
\text { cualquier } \\
\text { característica } \\
\text { esencial del } \\
\text { producto están } \\
\text { presentes, así } \\
\text { mismo no son } \\
\text { adicionados con } \\
\text { conservadores o } \\
\text { aditivos } \\
\text { similares. }\end{array}$ & $\begin{array}{l}\text { Son aquellos que } \\
\text { han sido } \\
\text { sometidos a } \\
\text { procesos } \\
\text { mecánicos } \\
\text { como: trituración, } \\
\text { molienda, } \\
\text { prensado o } \\
\text { también el uso de } \\
\text { hidrólisis o al } \\
\text { agregar aditivos, } \\
\text { enzimas u otro; } \\
\text { todo esto para la } \\
\text { manufacturación } \\
\text { de productos, } \\
\text { como los son los } \\
\text { productos } \\
\text { básicos } \\
\text { cocina. }\end{array}$ & $\begin{array}{l}\text { Estos utilizan } \\
\text { una } \\
\text { combinación } \\
\text { de los } \\
\text { mínimamente } \\
\text { y } \\
\text { medianamente } \\
\text { procesados, } \\
\text { con el fin de } \\
\text { obtener un } \\
\text { producto fácil y } \\
\text { rápido de } \\
\text { preparar. }\end{array}$ \\
\hline
\end{tabular}

Adaptado de Monteiro, C., Levy R., Moreira R., Rugani I., et al. Increasing consumption of ultra-processed foods and likely impact on human health. Public Health Nutr.

Estos alimentos tienen ciertas características que los hacen ser mayormente adquiridos por las personas, son 
de fácil accesibilidad, bajo costo, fácil preparación y con larga vida de anaquel. Actualmente vivimos en una época en la que la mayoría de las personas se encuentran laborando en actividades diferentes a la del hogar, por lo que muchas veces cuentan con poco tiempo para preparar sus alimentos, este es un factor importante que favorece que exista una sobreoferta de dichos tipos de alimentos.

En un estudio llevado a cabo en alimentos procesados y ultraprocesados para conocer si cumplen con los criterios del perfil de nutrientes de la Organización Panamericana de la Salud (OPS) en cuanto al contenido de sodio, azúcares añadidos, grasas totales y grasas saturadas por $100 \mathrm{~g} / \mathrm{ml}$ de producto mostró que en el grupo de alimentos procesados 4 subcategorías de 6: cereales, enlatados/envasados, lácteos y derivados, la razón de sodio fue mayor a 1 respecto al total de kcal del alimento, este resultado fue el mismo para los alimentos ultraprocesados en las categorías de: congelados, golosinas, bebidas, snacks, y deshidratados e instantáneos.

Se encontró también que los azúcares añadidos están fuera del criterio establecido por la OPS en alimentos como bebidas, dulces, enlatados/envasados y lácteos y derivados, mientras que para los alimentos ultra procesados son las golosinas, bebidas y deshidratados e instantáneos. En el caso de las grasas totales y grasas saturadas también sobrepasan las recomendaciones en los alimentos enlatados/envasados y lácteos y derivados en el caso de alimentos procesados y en los ultras procesados las golosina, snacks y deshidratados e instantáneos. $^{3}$

\section{OFERTA ALIMENTARIA Y SOBREOFERTA}

Podemos decir que una oferta alimentaria se refiere a la disponibilidad y accesibilidad que un alimento posee. Una sobreoferta representa a que existe un exceso en la oferta de un producto alimenticio, ahora bien, ¿porque se da una sobreoferta de los alimentos? Se presenta debido a la alta demanda de consumo de un alimento, pero esto dependerá de la accesibilidad alimentaria en los cuales se encuentran los gustos de la persona, ya sea por las características físicas, sensoriales o económicas que los hacen ser adquiridos, sin embargo, en el caso de los alimentos industrializados se ha analizado que estos alimentos causan adición, por lo que los hace ser mayormente consumidos por las personas.

Entre 2010 y 2018, las tiendas de conveniencia en México crecieron en un $142 \%$ mientras que los supermercados en un $64 \%$, se ha encontrado que una mayor densidad de tiendas de conveniencia se asocia con una mayor compra de alimentos ultraprocesados. ${ }^{8}$ Un estudio llevado a cabo en México encontró que los alimentos que tienen la contribución más alta de energía fueron los alimentos sin procesar o mínimamente procesados (54\%), seguido de los alimentos ultraprocesados (29.8\%), ingredientes culinarios procesados (10.2\%) y alimentos procesados (6.0\%). La contribución de energía de los alimentos ultraprocesados fue mayor en niños preescolares, en áreas urbanas, nivel socio económico medio y alto, así como en individuos con un nivel educativo más alto. ${ }^{6}$

\section{CONSUMO DE ALIMENTOS INDUSTRIALIZADOS ANTES DEL COVID-19}

El sistema alimentario abarca ciertas características, estas son la producción a escala, la existencia de un excesivo consumo de alimentos procesados los cuales aportan gran cantidad de energía y con una mínima cantidad de nutrientes y una alta cantidad de desperdicios, dichas características han llevado a que la población mexicana presente una inseguridad alimentaria esto debido a que existe una malnutrición por el consumo de estos tipos de alimentos que tienen un pobre perfil nutricional, sino al contrario conlleva a que por su alto aporte de energía se presenten problemas de sobrepeso y obesidad, los cuales son considerados como un problema de salud pública, estos alimentos también causan daño al medio ambiente por las sustancias y materiales que contienen. ${ }^{9}$

La industria alimentaria ha dominado el sistema alimentario con estrategias de marketing alentando a los consumidores a incluir más productos "listos para consumir" en su dieta, estos cambios han logrado que ahora este tipo de alimentos sean una importante fuente de energía y esta tendencia se está expandiendo globalmente. $^{10}$ Las familias mexicanas adquieren sus alimentos de acuerdo a sus gustos y economía, también por la oferta que estos presentan, así como los comportamientos alimentarios que observan en su entorno, esta última es sumamente importante debido a la cual se adquirirán los patrones alimenticios que pueden llegar a ser en un futuro perjudiciales para la salud si no se adoptan adecuados hábitos, especialmente en la infancia que es una etapa crucial para desarrollar dichos hábitos alimenticios. $^{9}$

Una de las características que han hecho que la población mexicana presente una dieta alta en grasa saturadas, azúcares añadidos y alimentos refinados bajos en fibra es el aumento del ingreso y la urbanización, lo que hace que exista una expansión en la producción y consumo de alimentos industrializados. ${ }^{9}$ En los entornos urbanos existe una mayor sobreoferta de alimentos industrializados debido a que la población realiza mayormente actividades cotidianas de traslado por sus diferentes actividades. ${ }^{11,12}$ Otro factor que ha influenciado en el deterioro de la salud de la población es la infraestructura insuficiente para beber agua limpia y las pocas medidas regulatorias que existen para ello. ${ }^{13}$ 
De acuerdo con la Encuesta Mensual de la Industria Manufacturera en la última década, los alimentos ultra procesados como carnes procesadas, botanas y galletas tuvieron una sobreoferta en relación con los alimentos básicos como leche y tortillas de maíz. ${ }^{1}$

\section{CONSUMO DE ALIMENTOS INDUSTRIALIZADOS DURANTE EL COVID-19}

En México las principales enfermedades crónicas son el sobrepeso, obesidad, diabetes mellitus e hipertensión arterial de acuerdo con datos de la ENSANUT 2018 y de acuerdo con la OPS en el año 2019 los principales alimentos industrializados mayormente consumidos fueron: refrescos con un $21.7 \%$, pasteles y postres con un $12 \%$ y snacks dulces y salados con un $10.4 \% .{ }^{14}$ Uno de los principales impulsores de esta crisis nutricional es el consumo de dichos alimentos. La obesidad se ve asociada a un estado inflamatorio crónico de baja intensidad que crea una respuesta inmune subóptima afectando de forma negativa el pronóstico de COVID19. ${ }^{15}$ En una conferencia de prensa el Subsecretario de Salud, dio a conocer que en México durante los últimos 10 años mueren en promedio 600 mil personas todos los años y que la mitad, es decir 300 mil, es debido a una mala alimentación. También añadió que no solo la persona es responsable de lo que consume, sino que también se debe a que existe una "monstruosa" y monumental sobreoferta de alimentos industrializados, indicó que al iniciar la epidemia de la COVID-19, México ya contaba con cuatro elementos importantes: el consumo excesivo de sal, grasa, azúcar y calorías totales, por los cuales hay que hacer énfasis en la importancia de trabajar en esta situación para mitigarlo. ${ }^{16}$ Actualmente el consumo de bebidas azucaradas provoca alrededor de 40 mil muertes de personas al año en México y esto se relaciona con el consumo de edulcorantes no calóricos, que provocan problemas como, enfermedades del corazón y ganancia de peso, esta última contribuye al sobrepeso/obesidad, diabetes e hipertensión y que nos vuelve mucho más vulnerables al COVID-19. De un total de 40 mil 842 muertes relacionadas por el consumo de bebidas azucaradas, 23 mil $433(57 \%)$ son a causa de diabetes mellitus, representando el $35.6 \%$ de todas las muertes por diabetes en México, 13 mil 517 (33\%) son causadas por enfermedades cardiovasculares, que corresponden al $12.7 \%$ de todas las muertes por esta causa, y 144 (-1\%) fueron ocasionadas por diferentes tipos de cáncer asociados a la obesidad.

La industria de bebidas asegura que para el 2024 habrá una reducción de calorías en sus productos, sin embargo lo que buscan realizar es sustituir los azúcares por edulcorantes no calóricos, con respecto a esto los diversos organismos y asociaciones, nacionales e internacionales recomiendan que los niños no consuman estos tipos de edulcorantes, debido a que a temprana edad el niño pueda desarrollar preferencias por los alimentos dulces y en un futuro se vea afectada su salud con hábitos alimenticios inadecuados. ${ }^{17}$

Es sumamente importante reconocer que mediante la pandemia del COVID-19 México sufrirá una mayor crisis económica, lo que como consecuencia reducirá la calidad de la dieta en la población mexicana, debido a la disminución en el consumo y suministro de verduras, frutas y alimentos de origen animal los cuales son principales fuentes de micronutrimentos y que son trascendentales en la dieta, en consecuencia se optaran dietas pobres en micronutrimentos, en los que predominarán los alimentos industrializados, esto también debido a que los programas públicos de distribución de alimentos en busca de ayudar a mitigar esta crisis, entregando estos tipos de alimentos no perecederos que afectaran mucho más dieta de los mexicanos.

Al existir una dieta baja en micronutrientes esenciales para el adecuado mantenimiento nutricional del cuerpo, el sistema inmune se verá debilitado y así mismo su capacidad para combatir la infección de COVID-19. ${ }^{18}$ Ahora bien lo que llama también la atención es un estudio realizado por el Public Health Nutrition que indico que el International Life Sciences Institute (ILSI), el cual es un ente que se dice promover la salud y el bienestar del público, es un grupo que trabaja junto con las industrias alimentarias para beneficio propio y la de esta empresas, recibiendo recursos monetarios por parte de estas industrias.$^{19}$

Los resultados preliminares de una encuesta llevada a cabo en población mexicana por el Instituto Nacional de Salud Pública de México en colaboración con el Programa Mundial de Alimentos cuyo objetivo fue entender el efecto de la contingencia por COVID-19 en el consumo y compra de alimentos de la población mexicana en el transcurso de la pandemia fueron los siguientes: ${ }^{20}$

La mayoría de la muestra es de un nivel socioeconómico y educativo alto. Sólo el $30 \%$ salía de casa por trabajo, la mitad de los individuos encuestados reportó que sus ingresos disminuyeron a partir de la pandemia por COVID-19, el 23\% aumentó su consumo de alimentos industrializados además de que en más de la mitad (53\%) la actividad física disminuyó. Otro estudio encontró en la población mexicana, un mayor aporte energético de los alimentos ultraprocesados se asoció con una menor calidad de la dieta en cuanto a la ingesta de nutrientes relacionados con enfermedades crónicas no transmisibles. ${ }^{21}$ 


\section{Conclusión}

La sobreoferta en el consumo de alimentos industrializados es sumamente preocupante, la modernización en el estilo de vida actual, el poco tiempo para realizar los alimentos por cuestiones de trabajo, está dirigiéndose cada vez más al consumo de estos alimentos, lo que nos hace pensar a donde nos dirigimos, cuáles serán las consecuencias en la salud en un futuro en la que actualmente ya nos vemos perjudicados. La obesidad, diabetes, hipertensión, y enfermedades cardiovasculares, seguirían siendo las principales causas de muerte en México.

Una dieta pobre en nutrientes, alta en calorías aunada a la pandemia del COVID-19 en el cual la población mexicana se ve más expuesta a consumir dichos alimentos que tienen larga vida en el anaquel y el sedentarismo nos dirige a este notable problema. La industria alimentaria aprovecha la sobreoferta que presenta sus productos y trata de alguna forma apoyar la crisis, informándole a sus consumidores por difusiones en los medios de comunicación su preocupación por lo vivido, aportando además apoyos económicos a las organizaciones que según promueven la salud de los mexicanos, cuando en realidad deberían de sujetarse a las normas que rigen las organizaciones encargadas al bienestar de la salud, apoyando por ejemplo el nuevo etiquetado frontal de alimentos de fácil interpretación, ya que como se presenta en este ensayo existen alimentos que no contemplan las cantidades adecuadas de azúcares, sodio y grasas, que son los principales causantes de las enfermedades no transmisibles. Hace falta también poner énfasis en la poca información de hábitos alimenticios adecuados en México, por lo que es importante contar con especialistas en esta área, que puedan educar a la población sobre elección correcta en los alimentos y preparaciones saludables con el objetivo de minimizar las consecuencias que se presentan al consumir estos tipos de alimentos procesados y así adquirir patrones alimenticios adecuados, cuidar nuestra salud y reducir el sobrepeso y obesidad en México.

\section{Referencias}

1. Aburto T., Pedraza L., Sáchez T., Batis C., et al. Discretionary Foods Have a High Contribution and Fruit, Vegetables, and Legumes Have a Low Contribution to the Total Energy Intake of the Mexican Population. The Journal of Nutrition. $\square$ Internet $\square 10$ de agosto 2016. $\square$ consultado el miércoles 03 de junio de $2020 \square$.

2. Pérez Izquierdo, Odette, Nazar Beutelspacher, Austreberta, Salvatierra Izaba, Benito, Pérez-Gil Romo, Sara Elena, Rodríguez, Luis, Castillo Burguete, María Teresa, \& Mariaca Méndez, Ramón. (2012). Frecuencia del consumo de alimentos industrializados modernos en la dieta habitual de comunidades mayas de Yucatán, México. Estudios sociales (Hermosillo, Son.), 20(39), 155-184. Recuperado en 20 de
diciembre
de
2020 ,
de http://www.scielo.org.mx/scielo.php?script=sci_arttext\&pid=S0188- $45572012000100006 \& \operatorname{lng}=\mathrm{es} \& \operatorname{tlng}=\mathrm{es}$.

3. Meza Miranda E, Nuñez BE, Maldonado O. Evaluación de la composición nutricional de alimentos procesados y ultraprocesados de acuerdo al perfil de alimentos de la organización panamericana de la salud, con énfasis en nutrientes críticos. Mem. Inst. Investig. Cienc. Salud. 2018; 16(1): 54-63

4. Muñoz J., Córdova J., Leveaga D., El índice de alimentación saludable de estudiantes de nuevo ingreso a una universidad de México. Nutr Hosp. $\square$ Internet $\square 2015 \square$ Consultado el lunes 01 de junio de $2020 \square$; 31(4):1582-1588. Disponible en: http://scielo.isciii.es/pdf/nh/v31n4/17 originalsindromemetabolico04.p df.

5. Favila H., López A., Precepción sobre el consumo de alimentos procesados entre familias de la ciudad de Toluca, México. Academia Journals $\square$ Intenet $\square 7,89$ de noviembre $2018 \square$ consultado el lunes 01 de junio de $2020 \square$.

6. Marrón-Ponce J., Sánchez-Pimienta T., da Costa M., Batis C. (2017) Energy contribution of NOVA food groups and sociodemographic determinants of ultra-processed food consumption in the Mexican population. Public Health Nutrition: 21(1), 87-93. doi: $10.1017 /$ S1368980017002129.

7. Monteiro, C., Levy R., Moreira R., Rugani I., et al. Increasing consumption of ultra-processed foods and likely impact on human health. Public Health Nutr. $\square$ Intenet $\square$ Junio 2011 $\square$ Consultado el lunes 01 de junio de $2020 \square ; 14(1): 5-13$.

8. Hernández- F M., Figueroa J., Colchero A. (2021) Association between density of stores and purchases of ultra-processed food and sugarsweetened beverages in Mexico. Health \& Place. https://doi.org/10.1016/j.healthplace.2021.102528.Hernández M., Unar M., Rivera J., Hacia un sistema alimentario promotor de dietas saludables y sostenibles. Instituto Nacional de Salud Pública. $\square$ Intenet $\square 2018 \square$ Consultado el martes 02 de junio de 2020 $\square$; 53-72.

9. Hernández M., Unar M., Rivera J., Hacia un sistema alimentario promotor de dietas saludables y sostenibles. Instituto Nacional de Salud Pública. $\square$ Intenet $\square 2018 \square$ Consultado el martes 02 de junio de 2020 $\square$; 53-72.

10. Marrón-Ponce J., Tolentino-Mayo L., Hernández-F M., Batis C. (2018) Trends in Ultra-Processed Food Purchases from 1984 to 2016 in Mexican Households. Nutrients 2019, 11, 45; doi:10.3390/nu11010045.

11. Boone J., Gordon P., Kiefe C., Shikany J., et al. Fast Food Restaurants and Food Stores Longitudinal Associations: With Diet in Young to Middle-aged Adults: The CARDIA. Study. Arch Intern Med. $\square$ Internet $\square 11$ de julio $2011 \square$ Consultado el martes 02 de junio de 2020 $;$ 171(13):1162-1170.

12. Ortiz L., Delgado G., Ana Hernández A. Cambios en factores relacionados con la transición alimentaria y nutricional en México. Gac Méd Méx. $\square$ Internet $\square 17$ de febrero de $2006 \square$ consultado el martes 02 de junio de 2020 $\square ; 142$ (3):181-193.

13. Barquera S., Rivera J. (2018) Obesity in Mexico: rapid epidemiological transition and food industry interference in health policies. Country Focuswww.thelancet.com/diabetes-endocrinology Vol 8 September 2020.

14. Alianza por la salud alimentaria. La pandemia de COVID-19 en medio de la epidemia de obesidad y diabetes en México. El poder del consumidor $\square$ Internet $\square 25$ de marzo de $2020 \square$ consultado el 03 de junio de $2020 \square$. 
15. White M., Nieto C., Barquera S. Good deeds and cheap marketing: The food industry in the times of COVID-19. $\square$ Internet $\square \square$ consultado el miércoles 03 de junio de $2020 \square$.

16. Aristegui Noticias Network. (2020, 20 diciembre). «Monstruosa» sobreoferta de alimentos industrializados incide en $300,000 \mathrm{mil}$ muertes al año en México: López-Gatell. Aristegui Noticias. https://aristeguinoticias.com/0404/mexico/monstruosa-sobreoferta-dealimentos-industrializados-incide-en-300000-mil-muertes-al-ano-enmexico-lopez-gatell/

17. El Poder del Consumidor A.C. (2020, 10 junio). Industria de bebidas azucaradas uno de los mayores atentados a la salud de los mexicanos El Poder del Consumidor. https://elpoderdelconsumidor.org/2020/05/industria-de-bebidasazucaradas-uno-de-los-mayores-atentados-a-la-salud-de-losmexicanos/?utm_campaign=meetedgar\&utm_medium=social\&utm_s ource $=$ meetedgar.com

18. International Food Policy Research Institute (IFPRI). (2020, 20 diciembre). The COVID-19 nutrition crisis: What to expect and how to protect. IFPRI : International Food Policy Research Institute. https://www.ifpri.org/blog/covid-19-nutrition-crisis-what-expect-andhow-protect

19. Arellano, S. (2020, 30 mayo). La pseudociencia y la pandemia de alimentos chatarra. Mexico Social. https://www.mexicosocial.org/lapseudociencia-y-la-pandemia-de-alimentos-chatarra/

20. Instituto Nacional de Salud Pública (2020). Estudio sobre el efecto de la contingencia por COVID-19 en el consumo y compra de alimentos. Disponible en: https://www.insp.mx/dieta-covid.

21. Marrón-Ponce J., Flores M., Cediel G., Monteiro C., Batis C. (2019) Associations between Consumption of Ultra-Processed Foods and Intake of Nutrients Related to Chronic Non-Communicable Diseases in Mexico. Journal of the academy of nutrition and dietetics, doi: 10.1016/j.jand.2019.04.020. 